\title{
The impact of general anesthesia, baseline ASPECTS, time to treatment, and IV tPA on intracranial hemorrhage after neurothrombectomy: pooled analysis of the SWIFT PRIME, SWIFT, and STAR trials
}

\author{
Radoslav Raychev (D) ,' Jeffrey L Saver, ${ }^{2}$ Reza Jahan, ${ }^{3}$ Raul G Nogueira, ${ }^{4}$ \\ Mayank Goyal, ${ }^{5}$ Vitor M Pereira, ${ }^{6}$ Jan Gralla, ${ }^{7}$ Elad I Levy (10 , ${ }^{8}$ Dileep R Yavagal, ${ }^{9}$ \\ Christophe Cognard, ${ }^{10}$ David S Liebeskind ${ }^{11}$
}

For numbered affiliations see end of article.

\section{Correspondence to} Dr. Radoslav Raychev, Department of Neurology and Comprehensive Stroke Center, University of California Los Angeles David Geffen School of Medicine, Los Angeles, CA 90095, USA; rudoray@gmail. com

Received 4 March 2019 Revised 8 May 2019 Accepted 12 May 2019 Published Online First 25 June 2019
Check for updates

(C) Author(s) (or their employer(s)) 2020. No commercial re-use. See rights and permissions. Published by BMJ.

To cite: Raychev R, Saver JL, Jahan R, et al.

J Neurolntervent Surg

2020;12:2-6.

\section{ABSTRACT}

Background Despite the proven benefit of neurothrombectomy, intracranial hemorrhage (ICH) remains the most serious procedural complication. The aim of this analysis was to identify predictors of different hemorrhage subtypes and evaluate their individual impact on clinical outcome.

Methods Pooled individual patient-level data from three large prospective multicenter studies were analyzed for the incidence of different ICH subtypes, including any $\mathrm{ICH}$, hemorrhagic transformation $(\mathrm{HT})$, parenchymal hematoma $(\mathrm{PH})$, subarachnoid hemorrhage $(\mathrm{SAH})$, and symptomatic intracranial hemorrhage (sICH). All patients $(n=389)$ treated with the Solitaire device were included in the analysis. A multivariate stepwise logistic regression model was used to identify predictors of each hemorrhage subtype.

Results General anesthesia and higher baseline Alberta Stroke Program Early CT score (ASPECTS) were associated with a lower probability of any ICH (OR $0.36, p=0.003)$, (OR 0.80, $p=0.032)$ and HT (OR 0.54, $p=0.023)$, (OR 0.78, $p=0.001)$, respectively. Longer time from onset to treatment was associated with a higher likelihood of HT (OR 1.08, $p=0.001)$ and PH (OR 1.11, $\mathrm{p}=0.015)$. Intravenous tissue plasminogen activator (IV-tPA) was also a strong predictor of PH (OR 7.63, $p=0.013$ ). Functional independence at 90 days (modified Rankin Scale (mRS) 0-2) was observed significantly less frequently in all hemorrhage subtypes except SAH. None of the patients who achieved functional independence at 90 days had $\mathrm{slCH}$.

Conclusions General anesthesia and smaller baseline ischemic core are associated with a lower probability of HT whereas IV-tPA and prolonged time to treatment increase the risk of $\mathrm{PH}$ after neurothrombectomy.

Trial registration numbers SWIFT-NCT01054560; post results, SWIFT PRIME-NCT01657461; post results, STAR-NCT01327989; post results.

\section{INTRODUCTION}

Hemorrhagic transformation (HT) in acute ischemic stroke (AIS) is known to occur as part of the natural history of cerebral infarction and its frequency and severity are increased by antithrombotics, anticoagulants, thrombolytics, and endovascular therapies. ${ }^{1}$
Given the overwhelming benefit of thrombectomy in selected patients with AIS due to anterior circulation large vessel occlusion (LVO), ${ }^{2-4}$ endovascular treatment (EVT) has been established as a standard of care in this patient population. ${ }^{56}$ However, intracranial hemorrhage $(\mathrm{ICH})$ remains the most dreaded complication of any acute revascularization therapy for AIS, and its potential occurrence requires careful risk versus benefit assessment in individual patients. Despite ample existing data demonstrating the hemorrhagic risk after intravenous and intra-arterial thrombolysis, little is known about the procedural and clinical factors associated with ICH incidence after contemporary thrombectomy with stent retriever. Identification of risk factors associated with $\mathrm{ICH}$ has the potential to further improve procedural safety and efficacy and guide patient selection in practice and future research. The clinical impact of various hemorrhage subtypes after EVT with stent retrievers has also not been well delineated. The purpose of this study was to identify the clinical, radiological, and procedural variables that may be predictive of various subtypes of ICH following mechanical thrombectomy.

\section{METHODS}

\section{Patient selection}

All patients who underwent EVT with the Solitaire device in the STAR $(n=202)$, SWIFT $(n=89)$, and SWIFT PRIME $(n=98)$ trials were included in this pooled analysis. All trials were large prospective multicenter studies involving patients with AIS with anterior circulation emergent large vessel occlusion (ELVO). The detailed designs, methods, and results of each individual trial have been previously published. ${ }^{7-10}$ All three study protocols were approved by the local ethics committee at each of the participating sites. All patients were enrolled after an informed consent form had been signed or country-specific requirements had been met for enrollment without explicit informed consent in emergency circumstances.

\section{Data collection}

Outcome data were adjudicated by an independent imaging/angiography core laboratory and a Clinical 
Events Committee (CEC). The core laboratory assessors were blinded to clinical outcome. Variables scored by the core laboratory and CEC were: location of occlusion, final revascularization grades, collaterals grading, Alberta Stroke Program Early CT score (ASPECTS), presence of ICH with corresponding subtypes, and other adverse events. In SWIFT and SWIFT PRIME, the assessors were blinded to study group assignments. The data were collected and provided by Medtronic Neurovascular. The company had no role in the design, conduct, analysis, or reporting of the present study.

\section{ICH categorization}

Different ICH subtypes were categorized by the core laboratory and CEC according to the European Cooperative Acute Stroke Study (ECASS) III definition as: (a) hemorrhagic transformation within the ischemic territory (HT), a category which included both hemorrhagic infarct (HI) and parenchymal hematoma (PH); and (b) symptomatic intracranial hemorrhage (sICH), defined as any $\mathrm{PH}$, subarachnoid hemorrhage $(\mathrm{SAH})$, or intraventricular hemorrhage (IVH) associated with death, or worsening of National Institutes of Health Stroke Scale score (NIHSS) by $\geq 4$ within 24 hours. ${ }^{11}$ Other imaging core laboratory-adjudicated ICH categories included: SAH, remote intracranial hemorrhage, and IVH. All the above referenced ICH subtypes were already categorized by the CEC and core laboratory for each individual trial and provided to us for the present analysis. From the pathophysiologic perspective, we analyzed HT (including all HI and $\mathrm{PH}$ and excluding SAH) as a separate category, which most likely represents post-reperfusion hemorrhage and less likely involves other potential mechanisms such as vessel dissection and wire perforation. The presence of any ICH was also categorized and analyzed separately. In the present study, all six separate hemorrhage subtypes (any ICH, HI, HT, PH, SAH, and sICH) were correlated with 15 candidate baseline clinical, imaging, and procedural variables (tables 1 and 2).

\section{Statistical analysis}

Standard descriptive statistics were employed, including means, SD and medians for continuous variables and frequency distributions for categorical variables. For unadjusted between-group comparisons, t-tests were used for continuous variables and

Table 1 Baseline variables included in the analysis

\begin{tabular}{|c|c|}
\hline Clinical variables & Procedural variables \\
\hline Age & General anesthesia \\
\hline NIHSS & Collateral grade* \\
\hline Hypertension & Number of device passes \\
\hline DM & Final $\mathrm{TICl}$ \\
\hline Hyperglycemia (BS >140 mg/dL) & Rescue therapy $\dagger$ \\
\hline \multicolumn{2}{|l|}{ Atrial fibrillation } \\
\hline \multicolumn{2}{|l|}{ INR } \\
\hline \multicolumn{2}{|l|}{ Platelet count } \\
\hline \multicolumn{2}{|l|}{ ASPECTS } \\
\hline \multicolumn{2}{|l|}{ IV-tPA } \\
\hline \multicolumn{2}{|c|}{$\begin{array}{l}\text { *Collateral grading was based on the ASITN scale. } \\
\text { †Any additional endovascular therapy after failed thrombectomy with Solitaire. } \\
\text { ASPECTS, Alberta Stroke Program Early CT score; DM, diabetes mellitus; INR, } \\
\text { international normalized ratio; IV-tPA, intravenous tissue plasminogen activator; } \\
\text { NIHSS, National Institutes of Health Stroke Scale; TICI, Thrombolysis in Cerebral } \\
\text { Infarction. }\end{array}$} \\
\hline
\end{tabular}

Table 2 Key baseline patient characteristics of the studied cohort

\begin{tabular}{ll}
\hline Characteristic & Result \\
\hline Female, \% (n/N) & $55 \%(214 / 389)$ \\
\hline Age, mean \pm SD (median) & $67.1 \pm 12.6(70)$ \\
\hline NIHSS, mean \pm SD (median) & $16.8 \pm 4.7(17)$ \\
\hline General anesthesia, \% (n/N) & $56 \%(217 / 389)$ \\
\hline Number of device passes, mean \pm SD (median) & $1.7 \pm 1.0(1)$ \\
\hline ASPECTS, mean \pm SD (median) & $8.3 \pm 1.7(9)$ \\
\hline IV-tPA, \% (n/N) & $66 \%(258 / 389)$ \\
\hline M1 occlusion, \% (n/N) & $67 \%(249 / 373)$ \\
\hline ICA occlusion, \% (n/N) & $19 \%(71 / 389)$ \\
\hline
\end{tabular}

ICA, internal carotid artery.

Fisher's exact test for categorical variables. Multivariate stepwise logistic regression was used to identify predictors of any $\mathrm{ICH}$ and individual hemorrhage subtypes using the list of candidate predictors cited in table 1 . Univariate analyses were run for each individual variable. Predictors with univariate $\mathrm{p}$ values $<0.20$ were included in the multivariable analyses. Stepwise selection with entry criteria of $\mathrm{p}<0.2$ and retention criteria of $\mathrm{p}<0.05$ were used to obtain the final regression model. Odds ratios were derived from these models and presented along with their 95\% CIs and corresponding p values. Fisher's exact test was used to compare the frequency of functional independence, defined as modified Rankin Scale (mRS) score of 0-2 at 90 days, among subgroups of patients with and without $\mathrm{ICH}$ and subtypes of ICH. Additional logistic regression, adjusted for baseline ASPECTS, was performed to compare ICH subtypes with 90-day functional independence. For all statistical analyses, two-tailed $\mathrm{p}$ values are presented, with values $<0.05$ considered statistically significant. Analyses were conducted in SAS Version 9.4 (SAS Institute, Cary, North Carolina, USA) and R Version 3.2 (R Foundation for Statistical Computing, Vienna, Austria). Table 2 represents the distribution of key baseline patient characteristics of the studied cohort.

\section{RESULTS}

Clinical and procedural predictors of ICH risk

Any ICH was observed in $84 / 389$ (21.6\%), HT in $75 / 389$ (19.3\%), HI in 54/389 (14\%), PH in 21/389 (5.4\%), SAH in $9 / 389(2.3 \%)$, and sICH only in $4 / 389(1 \%)$ of the analyzed patients. The most significant predictors of separate hemorrhage subtypes based on both univariate and multivariate analyses are shown in table 3. Both ASPECTS and general anesthesia (GA) had a strong inverse association with any ICH (ASPECTS: OR $0.80,95 \%$ CI 0.66 to $0.98, p=0.032$; GA: OR $0.36,95 \% \mathrm{CI}$ 0.18 to $0.71, \mathrm{p}=0.003$ ) and HT (ASPECTS: OR $0.78,95 \% \mathrm{CI}$ 0.68 to $0.91, \mathrm{p}=0.001$; GA: OR $0.54,95 \% \mathrm{CI} 0.31$ to 0.92 , $\mathrm{p}=0.023)$. Collateral grade was also inversely associated with any $\mathrm{ICH}$, but this association did not reach statistical significance $(p=0.057)$. Time to treatment was a significant hemorrhage predictor, with longer times increasing the risk of HT (OR $1.08,95 \% \mathrm{CI} 1.03$ to $1.12, \mathrm{p}=0.001)$ and $\mathrm{PH}(\mathrm{OR} 1.11,95 \% \mathrm{CI}$ 1.02 to $1.20, \mathrm{p}=0.015)$. IV-tPA use prior to thrombectomy was also a significant predictor of PH (OR 7.63, 95\% CI 1.52 to $17.35, \mathrm{p}=0.013)$. No significant predictors of $\mathrm{SAH}$ and $\mathrm{sICH}$ were identified, although a non-significant inverse association between sICH and platelet count was noted (OR 0.98, 95\% CI 0.96 to $1.0, \mathrm{p}=0.084)$. 
Table 3 Predictors of hemorrhage subtypes: pertinent findings from the multivariate analysis

\begin{tabular}{|c|c|c|c|c|}
\hline Predictor & OR & Lower $\mathrm{Cl}$ & Upper Cl & $P$ value \\
\hline \multicolumn{5}{|l|}{ Any ICH (HI, PH, SAH, and sICH) } \\
\hline ASPECTS & 0.80 & 0.66 & 0.98 & 0.032 \\
\hline General anesthesia & 0.36 & 0.18 & 0.71 & 0.003 \\
\hline Collateral grade & 0.71 & 0.50 & 1.01 & 0.057 \\
\hline \multicolumn{5}{|c|}{ Hemorrhagic transformation within the ischemic territory ( $\mathrm{HI}$ and $\mathrm{PH})$} \\
\hline ASPECTS & 0.78 & 0.68 & 0.91 & 0.001 \\
\hline General anesthesia & 0.54 & 0.31 & 0.92 & 0.023 \\
\hline Onset to groin puncture (per $15 \mathrm{~min}$ ) & 1.08 & 1.03 & 1.12 & 0.001 \\
\hline \multicolumn{5}{|l|}{ Parenchymal hematoma (PH) } \\
\hline IV r-tPA & 7.63 & 1.52 & 17.35 & 0.013 \\
\hline Onset to groin puncture (per $15 \mathrm{~min}$ ) & 1.11 & 1.02 & 1.20 & 0.015 \\
\hline \multicolumn{5}{|c|}{$\begin{array}{l}\text { ASPECTS , Alberta Stroke Program Early CT Score; } \mathrm{HI} \text {, hemorrhagic infarct; ICH, } \\
\text { intracranial hemorrhage; IV=tPA, intravenous tissue plasminogen activator; } \mathrm{PH} \text {, } \\
\text { parenchymal hematoma; SAH, subarachnoid hemorrhage; sICH, symptomatic } \\
\text { intracranial hemorrhage. }\end{array}$} \\
\hline
\end{tabular}

\section{Analysis of clinical outcome based on ICH subtype}

Among all patients treated with Solitaire in this dataset, 210/378 (55.6\%) were functionally independent at 90 days. The frequency of different hemorrhage subtypes seen within this group is shown in table 4. Additional adjusted analysis was performed to eliminate potential confounding between large ischemic core (low ASPECTS) and the occurrence of various ICH subtypes. Overall, patients with any ICH $(p<0.001)$, HT $(p<0.001)$, HI $(p=0.002)$, and PH $(p=0.002)$ were much less likely to have functional independence at 90 days than those who did not have hemorrhage. No significant difference in terms of functional independence was observed in patients with SAH $(p=0.46)$. None of the patients with sICH were independent at 90 days table 5 .

\section{DISCUSSION}

The findings of our study provide insights regarding pathophysiologic mechanisms and risk factors associated with various hemorrhagic subtypes after contemporary thrombectomy with Solitaire and their individual impact on functional independence.

\begin{tabular}{llll}
\hline \multicolumn{2}{l}{ Table 4} & Clinical outcome & \\
\hline ICH subtype & $\begin{array}{l}\text { Functional } \\
\text { independence* }\end{array}$ & $\begin{array}{l}\text { Functional } \\
\text { independence without ICH }\end{array}$ & P value \\
\hline $\begin{array}{l}\text { Any ICH (HT, PH, } \\
\text { SAH, sICH) }\end{array}$ & $32.1 \%(27 / 84)$ & $61.4 \%(183 / 298)$ & $<0.001$ \\
\hline HT (HI+PH) & $30.7 \%(23 / 75)$ & $60.9 \%(187 / 307)$ & $<0.001$ \\
\hline HI & $33.3 \%(19 / 57)$ & $58.8 \%(191 / 325)$ & $<0.001$ \\
\hline SAH & $44.4 \%(4 / 9)$ & $55.2 \%(206 / 373)$ & 0.74 \\
\hline PH & $19.0 \%(4 / 21)$ & $57.1 \%(206 / 361)$ & 0.001 \\
\hline SICH & $0.0 \%(0 / 4)$ & $55.6 \%(210 / 378)$ & 0.040 \\
\hline
\end{tabular}

Of the 389 patients in this study, ICH information was available in 382 and mRS information was available in 378 patients.

${ }^{*}$ Functional independence is defined as mRS $0-2$.

$\mathrm{HI}$, hemorrhagic infarct; $\mathrm{HT}$, hemorrhagic transformation; $\mathrm{ICH}$, intracranial hemorrhage; $\mathrm{PH}$, parenchymal hematoma; $\mathrm{SAH}$, subarachnoid hemorrhage; $\mathrm{sICH}$, symptomatic intracranial hemorrhage.
Table 5 Clinical outcome adjusted for baseline ASPECTS by logistic regression

\begin{tabular}{lll}
\hline ICH subtype & $\begin{array}{l}\text { Adjusted odds ratio (aOR) for } \\
\text { mRS 0-2 outcome }(95 \% \mathrm{Cl})\end{array}$ & P value \\
\hline Any ICH (HT, PH, SAH, sICH) & $0.31(0.19,0.53)$ & $<0.001$ \\
\hline HT (HI+PH) & $0.30(0.17,0.52)$ & $<0.001$ \\
HI & $0.38(0.21,0.70)$ & 0.002 \\
SAH & $0.60(0.16,2.30)$ & 0.46 \\
PH & $0.18(0.06,0.54)$ & 0.002 \\
sICH $\dagger$ & NA (NA) & NA
\end{tabular}

$\mathrm{ICH}$ on $\mathrm{mRS}$ 0-2 outcomes while controlling for (and therefore independent of) any effect of baseline ASPECTS on clinical outcome. Results are concordant with univariate modeling unadjusted for ASPECTS (table 4).

*The analysis shown above displays the adjusted odds ratio (aOR) for the occurrence of mRS 0-2 in the presence of each ICH subtype shown, adjusted for ASPECTS score at baseline. The adjustment for ASPECTS is included to avoid confounding between low ASPECTS and subsequent ICH. The aOR shown represents the effect of

†Adjusted modeling could not be performed due to sparse data $(n=4$ in sICH group).

\section{Impact of general anesthesia}

Our study is the first to report an inverse association between GA and the occurrence of ICH in the setting of neurothrombectomy. Although GA did not impact the most severe hemorrhage subtypes (sICH and $\mathrm{PH}$ ), these findings provide signals about its protective effect against hemorrhage that warrant further exploration. While no definitive explanation regarding the relationship between GA and ICH can be drawn from our data, there are several theoretical possibilities: (1) less patient motion in the setting of GA with more precise intracranial navigation and lower chance of microvascular avulsion; (2) protective effect of anesthetic medications against cerebral ischemic reperfusion injury ${ }^{12-15}$; (3) reduced pain and agitation with lower chance of blood pressure surges and uncontrolled hypertension, which is an independent risk of HT. ${ }^{16}$ In addition, hypotension that frequently occurs with GA during thrombectomy may also protect the ischemic tissue against HT after reperfusion. However, it is also important to emphasize that hypotension can be deleterious to the cerebral collateral circulation prior to revascularization. Profound hemodynamic and ventilation changes in the setting of GA have been associated with worse outcome after thrombectomy, ${ }^{17}$ and are likely key factors leading to an overall worse outcome seen in multiple retrospective case series and post hoc analyses correlating GA with worse clinical outcome. ${ }^{18-23}$ In contrast, three single-center prospective randomized studies exploring the difference between GA and conscious sedation (CS) demonstrated equivalence in their primary outcomes. ${ }^{24-26}$ Furthermore, two of these three recent trials showed superiority in terms of functional independence of GA over CS as a secondary outcome. ${ }^{24} 26$ All three trials had specific GA protocols with well-defined ventilation and blood pressure targets designed to minimize the risk of collateral failure prior to endovascular revascularization. Accordingly, two recent meta-analyses involving newer thrombectomy devices demonstrated inconclusive results regarding the clinical benefit of either procedural method of sedation. ${ }^{27} 28$ Given the prior confounding data, the most recent guidelines for early management of AIS specifically outlined the need for further randomized data to identify the best anesthetic technique. ${ }^{6}$ Our findings of the protective effect of GA against ICH further contribute to the existing clinical equipoise regarding the optimal method of sedation during EVT. 


\section{Impact of baseline ASPECTS}

The size of the baseline ischemic core is a well-established predictor of hemorrhagic risk with intravenous and endovascular reperfusion. $^{29-33}$ The strong association of baseline ASPECTS with any $\mathrm{ICH}$ and $\mathrm{HT}$ in our study reaffirms these previous data and further establishes the ischemic volume as one of the most important factors influencing hemorrhage after reperfusion. Cerebral collateral circulation, which is inversely proportional to the ischemic core volume, is another important and wellknown variable associated with an increased risk of HT after intra-arterial therapies for $\mathrm{AIS}^{33-35}$ and also was linked to $\mathrm{ICH}$ in our study. Both variables demonstrate the increased susceptibility of the ischemic core tissue to HT after thrombectomy.

\section{Impact of time to treatment}

Prolonged time from onset to treatment is one of the main determinants of clinical outcome in all AIS therapies. ${ }^{36-38}$ The wellestablished pathophysiologic paradigm is that progression of the ischemic core lesion in the setting of LVO is highly dependent on the temporal dynamic evolution of collateral circulation, which fails over time. ${ }^{39}$ As discussed above, ischemic core volume and collaterals are potent predictors of HT. Ischemia-induced blood-brain barrier disruption, ${ }^{40}$ activation of metalloproteinases, ${ }^{41}$ and formation of free radicals ${ }^{42}$ rendering the ischemic brain susceptible to hemorrhage are proportionally related to the degree and size of the ischemic changes and occur at maximum intensity within 6-24 hours after onset. ${ }^{43} 44$ Thus, the association between time to treatment and hemorrhagic risk in our study is not surprising as it likely represents the increased vulnerability of the ischemic brain tissue to reperfusion hemorrhage in the setting of prolonged ischemia. Similar findings have been reported in previous studies: prolonged time from onset to EVT has been associated with an increased risk of basal ganglionic hemorrhage and sICH. ${ }^{32} 33$ Experience with faster intravenous thrombolysis further supports this notion: in a study involving over 70000 patients, reduction of door-to-needle time for IV-tPA administration after implementation of national quality initiatives resulted in a significant decrease in the incidence of $\mathrm{ICH}^{45}$

\section{Impact of IV-tPA}

Similar to GA, the data regarding the benefit of bridging IV-tPA therapy in the setting of EVT for ELVO is a topic of ongoing controversy and debate. ${ }^{46}$ The main reason supporting its use is improved recanalization and potential avoidance of thrombectomy, particularly in patients with distal and smaller clots. ${ }^{47}$ However, the small chance of recanalization after early administration of IV-tPA for LVO in comprehensive or thrombectomy-ready centers may be obviated by rapid endovascular revascularization. A small single-center study demonstrated equivalent outcomes with EVT alone versus IV-tPA/EVT bridging therapy. ${ }^{48}$ Another consideration against bridging therapy in ELVO patients is the increased cost of IV-tPA. ${ }^{49}$ In addition, the coagulopathy induced by the tPA clearly accentuates the hemorrhagic risk after reperfusion. ${ }^{50} 51$ These post-thrombolytic hemostasis changes have been correlated with cardioembolic infarcts, ${ }^{52}$ which comprise most of the LVO etiology and further raise the concern of an augmented risk of hemorrhage after EVT. Unlike the other predictors, IV-tPA was uniquely associated with $\mathrm{PH}$ in our study. $\mathrm{PH}$ has been considered as a separate entity due to its highest impact on clinical outcome among all hemorrhagic subtypes, ${ }^{53}$ which further emphasizes the potentially hazardous effect of bridging IV-tPA therapy in conjunction with EVT. A similar finding of a higher PH risk with IV-tPA from 'real-world' non-randomized retrospective data involving older generation devices was reported by Nogueira et al. ${ }^{54}$ As such, our findings contribute to the existing data of the increased hemorrhagic risk of IV-tPA, reaffirm the controversy regarding the utility of IV-tPA in the setting of ELVO, and support the need for a well-powered randomized multicenter trial comparing bridging therapy versus EVT alone.

\section{Impact of ICH subtypes on outcome}

We explored the incidence of functional independence (mRS $0-2)$ with $\mathrm{ICH}$ versus the incidence of functional independence without ICH for each hemorrhagic subtype. Although this analysis involved simple univariate comparisons, it provides important information regarding the potential impact of each type of hemorrhage on clinical outcome. All hemorrhage subtypes were associated with a lower likelihood of independent clinical outcome, except for SAH. This finding replicates prior data, emphasizing that the imaging finding of SAH alone without parenchymal involvement and/or clinical deterioration may not have a significant long-term clinical impact. ${ }^{55-57}$

None of the currently used definitions of sICH optimally predict functional outcome. ${ }^{58}$ The data regarding the long-term clinical effect of different radiographic subtypes are also unclear. While $\mathrm{PH}$ has been cited as the most potentially hazardous to clinical outcome, ${ }^{53} \mathrm{HI}$ has been considered a relatively 'benign' entity and even correlated with improved outcome as a marker of early reperfusion after IV-tPA in some studies. ${ }^{59}$ Conversely, more detailed analyses have identified asymptomatic HI-2 as an independent predictor of poor outcome after IV-tPA and EVT. $^{5460}$ Our data support the notion that any ICH, HI, and HT can have a potential impact on functional outcome, even if considered asymptomatic by trial definitions. However, our findings also highlight that the stringent definition of sICH used in this dataset (any PH, SAH, or IVH associated with death or worsening of NIHSS score by $\geq 4$ within 24 hours) and recent thrombectomy trials has the strongest impact on functional outcome as none of the patients with sICH achieved mRS 0-2 at 90 days.

\section{Limitations}

Our study has several limitations. The main limitation is the relatively small number of symptomatic hemorrhages in the dataset as the patient population included in the three trials is highly selected and may not represent 'real-world' experience. Another important drawback is the difference in the design of the three studies, conducted in three difference continents over a time span of 4 years. Although all patients were treated with the same device, these factors introduce potential heterogeneity in the analyzed data due to differences in patient population and neurointerventional practices. In addition, our clinical outcome data were derived on the basis of analyses adjusted only for baseline ASPECTS, instead of multivariate regression, focusing only on individual hemorrhage effect and excluding other important clinical and procedural variables. However, the goal of the outcome analysis was to analyze the clinical effect of each $\mathrm{ICH}$ subtype compared with all other hemorrhages rather than identifying overall predictors of clinical outcome.

\section{CONCLUSIONS}

Detailed knowledge regarding the factors associated with hemorrhage occurrence after endovascular reperfusion for AIS may improve procedural safety and outcomes. Our data demonstrate that lower baseline ASPECTS, delayed time to treatment, and 
bridging IV-tPA are significant predictors of ICH after thrombectomy with Solitaire. This report also provides novel evidence that GA minimizes the peri-procedural hemorrhagic risk and may be a safer alternative to CS in the proper institutional settings. These findings could be applied for better patient selection in current practice and indicate the need for randomized data addressing the benefit of bridging therapy with IV thrombolysis and the optimal method of sedation in endovascular treatment of AIS.

\section{Author affiliations}

Department of Neurology and Comprehensive Stroke Center, University of California Los Angeles David Geffen School of Medicine, Los Angeles, California, USA ${ }^{2}$ Division of Interventional Neuroradiology, University of California Los Angeles Medical Center, Los Angeles, California, USA

${ }^{3}$ Grady Memorial Hospital Marcus Stroke \& Neuroscience Center, Atlanta, Georgia, USA

${ }^{4}$ Department of Neurology, Emory University School of Medicine, Atlanta, Georgia, USA

${ }^{5}$ Departments of Radiology and Clinical Neurosciences, University of Calgary, Calgary, Alberta, Canada

${ }^{6}$ Division of Neuroradiology, Medical Imaging, University Health Network - Toronto Western Hospital, Toronto, Ontario, Canada

${ }^{7}$ Division of Neurosurgery, Department of Surgery, University Health Network Toronto Western Hospital, Toronto, Ontario, Canada

${ }^{8}$ Department of Neurosurgery, State University of New York, Buffalo, New York, USA

${ }^{9}$ University of Miami and Jackson Memorial Hospitals, Miami, Florida, USA

${ }^{10}$ Department of Diagnostic and Therapeutic Neuroradiology, University Hospital of Toulouse, Toulouse, France

"Department of Neurology, University of California Los Angeles, Neurovascular Imaging Research Core, Los Angeles, California, USA

Correction notice Since this article was first published online first, a change has been made to the conclusion section of the abstract.

Contributors The main author interpreted the data, drafted the original manuscript version, reviewed all suggestions provided by all co-authors, approved the final version, and agreed to be accountable for all aspects of the work in ensuring that questions related to the accuracy or integrity of any part of the work are appropriately investigated and resolved. All co-authors provided substantial contribution to the interpretation of the provided data. All co-authors also contributed with revisions to the original draft, approved the final version of the manuscript, and agreed to be accountable for all aspects of the work.

Funding All three trials (STAR, SWIFT, and SWIFT PRIME) were funded by Medtronic Neurovascular.

Competing interests All co-authors are consultants for Medtronic Neurovascular. Patient consent for publication Not required.

Provenance and peer review Not commissioned; externally peer reviewed.

ORCID iDs

Radoslav Raychev http://orcid.org/0000-0001-8463-0664

Elad I Levy http://orcid.org/0000-0002-6208-3724

\section{REFERENCES}

1 Álvarez-Sabín J, Maisterra O, Santamarina E, et al. Factors influencing haemorrhagic transformation in ischaemic stroke. Lancet Neurol 2013:12:689-705.

2 Goyal M, Menon BK, van Zwam WH, et al. Endovascular thrombectomy after large-vessel ischaemic stroke: a meta-analysis of individual patient data from five randomised trials. Lancet 2016;387:1723-31.

3 Nogueira RG, Jadhav AP, Haussen DC, et al. Thrombectomy 6 to 24 Hours after Stroke with a Mismatch between Deficit and Infarct. N Eng/ J Med 2018;378.

4 Albers GW, Marks MP, Kemp S, et al. Thrombectomy for Stroke at 6 to 16 Hours with Selection by Perfusion Imaging. N Eng/ J Med 2018;378:708-18.

5 Powers WJ, Derdeyn CP, Biller J, et al. American Heart Association Stroke Council. 2015 American Heart Association/American Stroke Association Focused Update of the 2013 Guidelines for the Early Management of Patients With Acute Ischemic Stroke Regarding Endovascular Treatment: A Guideline for Healthcare Professionals From the American Heart Association/American Stroke Association. Stroke 2015;46:3020-35.

6 Powers WJ, Rabinstein AA, Ackerson T, et al. American Heart Association Stroke Council. 2018 Guidelines for the Early Management of Patients With Acute Ischemic Stroke: A Guideline for Healthcare Professionals From the American Heart Association/ American Stroke Association. Stroke 2018;49.

7 Pereira VM, Gralla J, Davalos A, et al. Prospective, multicenter, single-arm study of mechanical thrombectomy using Solitaire Flow Restoration in acute ischemic stroke. Stroke 2013;44:2802-7.
8 Saver JL, Goyal M, Bonafe A, et al. Solitaire ${ }^{\mathrm{TM}}$ with the Intention for Thrombectomy as Primary Endovascular Treatment for Acute Ischemic Stroke (SWIFT PRIME) trial: protocol for a randomized, controlled, multicenter study comparing the Solitaire revascularization device with IV tPA with IV IPA alone in acute ischemic stroke. Int Stroke 2015;10:439-48.

9 Saver JL, Goyal M, Bonafe A, et al. Stent-retriever thrombectomy after intravenous t-PA vs. t-PA alone in stroke. N Eng/ J Med 2015;372:2285-95.

10 Saver JL, Jahan R, Levy El, et al. Solitaire flow restoration device versus the Merci Retriever in patients with acute ischaemic stroke (SWIFT): a randomised, parallelgroup, non-inferiority trial. Lancet 2012;380:1241-9.

11 Hacke W, Kaste M, Bluhmki E, et al. Thrombolysis with alteplase 3 to 4.5 hours after acute ischemic stroke. N Engl J Med 2008;359:1317-29.

12 Cheon SY, Kim SY, Kam EH, et al. Isoflurane preconditioning inhibits the effects of tissue-type plasminogen activator on brain endothelial cell in an in vitro model of ischemic stroke. Int J Med Sci 2017:14:425-33.

13 Wang H, Zheng S, Liu M, et al. The Effect of Propofol on Mitochondrial Fission during Oxygen-Glucose Deprivation and Reperfusion Injury in Rat Hippocampal Neurons. PLoS One 2016;11:e0165052.

$14 \mathrm{Ji}$ FT, Liang JJ, Miao LP, et al. Propofol post-conditioning protects the blood brain barrier by decreasing matrix metalloproteinase- 9 and aquaporin- 4 expression and improves the neurobehavioral outcome in a rat model of focal cerebral ischemiareperfusion injury. Mol Med Rep 2015;12:2049-55.

15 Li J, Yu W, Li XT, et al. The effects of propofol on mitochondrial dysfunction following focal cerebral ischemia-reperfusion in rats. Neuropharmacology 2014;77:358-68.

$16 \mathrm{Ko} \mathrm{Y,} \mathrm{Park} \mathrm{JH,} \mathrm{Yang} \mathrm{MH,} \mathrm{et} \mathrm{al.} \mathrm{The} \mathrm{significance} \mathrm{of} \mathrm{blood} \mathrm{pressure} \mathrm{variability} \mathrm{for}$ the development of hemorrhagic transformation in acute ischemic stroke. Stroke 2010;41:2512-8

17 Jagani M, Brinjikji W, Rabinstein AA, et al. Hemodynamics during anesthesia for intraarterial therapy of acute ischemic stroke. J Neurointerv Surg 2016;8:883-8.

18 Abou-Chebl A, Lin R, Hussain MS, et al. Conscious sedation versus general anesthesia during endovascular therapy for acute anterior circulation stroke: preliminary results from a retrospective, multicenter study. Stroke; a journal of cerebral circulation 2010;41:1175-9.

19 Brinjikji W, Murad MH, Rabinstein AA, et al. Conscious sedation versus general anesthesia during endovascular acute ischemic stroke treatment: a systematic review and meta-analysis. AJNR Am J Neuroradiol 2015;36:525-9.

20 Davis MJ, Menon BK, Baghirzada LB, et al. Calgary Stroke Program. Anesthetic management and outcome in patients during endovascular therapy for acute stroke. Anesthesiology 2012:116:396-405.

21 John S, Thebo U, Gomes J, et al. Intra-arterial therapy for acute ischemic stroke under general anesthesia versus monitored anesthesia care. Cerebrovasc Dis 2014;38:262-7.

22 Just C, Rizek P, Tryphonopoulos P, et al. Outcomes of General Anesthesia and Conscious Sedation in Endovascular Treatment for Stroke. Can I Neuro Sci 2016;43:655-8.

23 van den Berg LA, Koelman DL, Berkhemer OA, et al. MR CLEAN pretrial study groupParticipating centers. Type of anesthesia and differences in clinical outcome after intra-arterial treatment for ischemic stroke. Stroke 2015;46:1257-62.

24 Schönenberger S, Uhlmann L, Hacke W, et al. Effect of Conscious Sedation vs General Anesthesia on Early Neurological Improvement Among Patients With Ischemic Stroke Undergoing Endovascular Thrombectomy. JAMA 2016;316:1986-96.

25 Lowhagen Henden P, Rentzos A, Karlsson JE, et al. General Anesthesia Versus Conscious Sedation for Endovascular Treatment of Acute Ischemic Stroke: The AnStroke Trial (Anesthesia During Stroke). Stroke 2017:48:1601-7.

26 Simonsen CZ, Yoo AJ, Sørensen LH, et al. Effect of General Anesthesia and Conscious Sedation During Endovascular Therapy on Infarct Growth and Clinical Outcomes in Acute Ischemic Stroke. JAMA Neurol 2018;75:470.

27 Brinjikji W, Pasternak J, Murad MH, et al. Anesthesia-Related Outcomes for Endovascular Stroke Revascularization: A Systematic Review and Meta-Analysis. Stroke 2017:48:2784-91.

28 Ilyas A, Chen CJ, Ding D, et al. In Reply to the Letter to the Editor Regarding "Endovascular Mechanical Thrombectomy for Acute Ischemic Stroke Under General Anesthesia Versus Conscious Sedation: A Systematic Review and Meta-Analysis". World Neurosurg 2018;115:489.

29 Barber PA, Demchuk AM, Zhang J, et al. Validity and reliability of a quantitative computed tomography score in predicting outcome of hyperacute stroke before thrombolytic therapy. ASPECTS Study Group. Alberta Stroke Programme Early CT Score. Lancet 2000;355:1670-4.

30 Souza LC, Payabvash S, Wang Y, et al. Admission CT perfusion is an independent predictor of hemorrhagic transformation in acute stroke with similar accuracy to DWI. Cerebrovasc Dis 2012:33:8-15.

31 Campbell BCV, Christensen S, Parsons MW, et al. Advanced imaging improves prediction of hemorrhage after stroke thrombolysis. Ann Neurol 2013;73:510-9.

32 Raychev R, Jahan R, Liebeskind D, et al. SWIFT Trial Investigators. Determinants of Intracranial Hemorrhage Occurrence and Outcome after Neurothrombectomy Therapy: Insights from the Solitaire FR With Intention For Thrombectomy Randomized Trial. AJNR Am J Neuroradiol 2015;36:2303-7. 
33 Hao Y, Yang D, Wang H, et al. ACTUAL Investigators (Endovascular Treatment for Acute Anterior Circulation Ischemic Stroke Registry). Predictors for Symptomatic Intracranial Hemorrhage After Endovascular Treatment of Acute Ischemic Stroke. Stroke 2017:48:1203-9.

34 Christoforidis GA, Karakasis C, Mohammad Y, et al. Predictors of hemorrhage following intra-arterial thrombolysis for acute ischemic stroke: the role of pial collateral formation. AJNR Am J Neuroradiol 2009;30:165-70.

35 Bang OY, Saver JL, Kim SJ, et al. UCLA-Samsung Stroke Collaborators. Collateral flow averts hemorrhagic transformation after endovascular therapy for acute ischemic stroke. Stroke 2011:42:2235-9.

36 Saver JL, Fonarow GC, Smith EE, et al. Time to treatment with intravenous tissue plasminogen activator and outcome from acute ischemic stroke. JAMA 2013;309:2480-8

37 Sheth SA, Jahan R, Gralla J, et al. Time to endovascular reperfusion and degree of disability in acute stroke. Ann Neurol 2015;78:584-93.

38 Saver JL, Goyal M, van der Lugt A, et al. HERMES Collaborators. Time to Treatment With Endovascular Thrombectomy and Outcomes From Ischemic Stroke: A Metaanalysis. JAMA 2016;316:1279-88.

39 Liebeskind DS. Collateral perfusion: time for novel paradigms in cerebral ischemia. Int J Stroke 2012;7:309-10.

40 del Zoppo GJ, von Kummer R, Hamann GF. Ischaemic damage of brain microvessels: inherent risks for thrombolytic treatment in stroke. J Neurol Neurosurg Psychiatry 1998;65:1-9

41 Fujimura M, Gasche Y, Morita-Fujimura Y, et al. Early appearance of activated matrix metalloproteinase- 9 and blood-brain barrier disruption in mice after focal cerebral ischemia and reperfusion. Brain Res 1999;842:92-100.

42 Peters $\mathrm{O}, \mathrm{Back} \mathrm{T}$, Lindauer $\mathrm{U}$, et al. Increased formation of reactive oxygen species after permanent and reversible middle cerebral artery occlusion in the rat. J Cereb Blood Flow Metab 1998;18:196-205.

43 Merali Z, Huang K, Mikulis D, et al. Evolution of blood-brain-barrier permeability after acute ischemic stroke. PLoS One 2017;12:e0171558.

44 Sandoval KE, Witt KA. Blood-brain barrier tight junction permeability and ischemic stroke. Neurobiol Dis 2008;32:200-19.

45 Fonarow GC, Zhao X, Smith EE, et al. Door-to-needle times for tissue plasminogen activator administration and clinical outcomes in acute ischemic stroke before and after a quality improvement initiative. JAMA 2014;311:1632-40.

46 Chandra RV, Leslie-Mazwi TM, Mehta BP, et al. Does the use of IV tPA in the current era of rapid and predictable recanalization by mechanical embolectomy represent good value?: Table 1. J Neurointerv Surg 2016;8:443-6.

47 Mishra SM, Dykeman J, Sajobi TT, et al. Early reperfusion rates with IV tPA are determined by CTA clot characteristics. AJNR Am J Neuroradiol 2014;35:2265-72.
48 Leker RR, Pikis S, Gomori JM, et al. Is Bridging Necessary? A Pilot Study of Bridging versus Primary Stentriever-Based Endovascular Reperfusion in Large Anterior Circulation Strokes. J Stroke Cerebrovasc Dis 2015;24:1163-7.

49 Rai AT, Boo S, Buseman C, et al. Intravenous thrombolysis before endovascular therapy for large vessel strokes can lead to significantly higher hospital costs without improving outcomes. J Neurointerv Surg 2018;10:17-21.

50 Trouillas P, Derex L, Philippeau F, et al. Early fibrinogen degradation coagulopathy is predictive of parenchymal hematomas in cerebral rt-PA thrombolysis: a study of 157 cases. Stroke 2004;35:1323-8.

51 Sun X, Berthiller J, Trouillas P, et al. Early fibrinogen degradation coagulopathy: A predictive factor of parenchymal hematomas in cerebral rt-PA thrombolysis. J Neurol Sci 2015;351(1-2):109-14.

52 Sun X, Berthiller J, Derex L, et al. Post-thrombolysis haemostasis changes after rt-PA treatment in acute cerebral infarct. Correlations with cardioembolic aetiology and outcome. J Neurol Sci 2015:349(1-2):77-83.

53 von Kummer R, Broderick JP, Campbell BC, et al. The Heidelberg Bleeding Classification: Classification of Bleeding Events After Ischemic Stroke and Reperfusion Therapy. Stroke 2015;46:2981-6.

54 Nogueira RG, Gupta R, Jovin TG, et al. Predictors and clinical relevance of hemorrhagic transformation after endovascular therapy for anterior circulation large vesse occlusion strokes: a multicenter retrospective analysis of 1122 patients. J Neurointerv Surg 2015;7:16-21.

55 Shi ZS, Liebeskind DS, Loh Y, et al. UCLA Endovascular Stroke Therapy Investigators. Predictors of subarachnoid hemorrhage in acute ischemic stroke with endovascular therapy. Stroke 2010;41:2775-81.

56 Nikoubashman 0, Reich A, Pjontek R, et al. Postinterventional subarachnoid haemorrhage after endovascular stroke treatment with stent retrievers. Neuroradiology 2014;56:1087-96.

57 Yoon W, Jung MY, Jung SH, et al. Subarachnoid hemorrhage in a multimodal approach heavily weighted toward mechanical thrombectomy with solitaire stent in acute stroke. Stroke 2013;44:414-9.

58 Gumbinger C, Gruschka P, Böttinger M, et al. Improved prediction of poor outcome after thrombolysis using conservative definitions of symptomatic hemorrhage. Stroke 2012;43:240-2.

59 Molina CA, Alvarez-Sabin J, Montaner J, et al. Thrombolysis-related hemorrhagic infarction: a marker of early reperfusion, reduced infarct size, and improved outcome in patients with proximal middle cerebral artery occlusion. Stroke 2002;33:1551-6.

60 Dzialowski I, Pexman JH, Barber PA, et al. Asymptomatic hemorrhage after thrombolysis may not be benign: prognosis by hemorrhage type in the Canadian alteplase for stroke effectiveness study registry. Stroke 2007;38:75-9.

61 Higashida RT, Furlan AJ, et al. Trial Design and Reporting Standards for Intra-Arterial Cerebral Thrombolysis for Acute Ischemic Stroke. Stroke 2003;34:e109-37. 
Correction: The impact of general anesthesia, baseline ASPECTS, time to treatment, and IV tPA on intracranial hemorrhage after neurothrombectomy: pooled analysis of the SWIFT PRIME, SWIFT, and STAR trials

Raychev R, Saver JL, Jahan R, et al. The impact of general anesthesia, baseline ASPECTS, time to treatment, and IV tPA on intracranial hemorrhage after neurothrombectomy: pooled analysis of the SWIFT PRIME, SWIFT, and STAR trials. J NeuroIntervent Surg 2020;12:2-6. doi: 10.1136/neurintsurg-2019-014898.

This article has been corrected since it was published Online First. The affiliation for Dr Jan Gralla has been updated to the University Institute of Diagnostic and Interventional Neuroradiology, University Hospital Bern, Inselspital, University of Bern, Switzerland.

(c) Author(s) (or their employer(s)) 2021. No commercial re-use. See rights and permissions. Published by BMJ.

J Neurolntervent Surg 2021;13:e21. doi:10.1136/neurintsurg-2019-014898corr1

D) Check for updates 